\title{
Association between the BDNF Val66Met Polymorphism and Chronicity of Depression
}

\author{
Yujin Lee1, Shinn Won Lim², Soo Yeon Kim², Jae Won Chung', Jinwoo Kim¹, Woojae Myung1, \\ Jihae Song ${ }^{1}$, Seonwoo Kim ${ }^{3}$, Bernard J Carroll ${ }^{4}$ and Doh Kwan Kim ${ }^{1,2} \bowtie$ \\ ${ }^{1}$ Department of Psychiatry, Samsung Medical Center, Sungkyunkwan University School of Medicine, Seoul, Republic of Korea \\ ${ }^{2}$ Center for Clinical Research, Sungkyunkwan University School of Medicine, Seoul, Republic of Korea \\ ${ }^{3}$ Biostatistics Team, Samsung Biomedical Research Institute, Sungkyunkwan University School of Medicine, Seoul, Republic of Korea \\ ${ }^{4}$ Pacific Behavioral Research Foundation, Carmel, CA, USA
}

Objective Both clinical and biological factors influence the course of depressive disorders. This study tested for associations between the brain-derived neurotrophic factor (BDNF) gene at the Val66Met locus and the course of major depressive disorder (MDD).

Methods Three hundred ten Korean subjects (209 patients, 101 controls) were genotyped for rs6265 at nucleotide 196 (G/A), which produces an amino acid substitution at codon 66 (Val66Met) of the gene for BDNF. Course of illness was evaluated both by chronicity of current episode (episode duration $>24$ months) and by the lifetime history of recurrences.

Results Patients with the Met/Met BDNF genotype had a significantly higher rate of chronic depression than all others. There was a significant dose effect of the Met allele on chronicity. Compared with the Val/Val genotype, the relative risk of chronicity was 1.67 for the Val/ Met genotype, and 2.58 for the Met/Met genotype. Lifetime history of recurrent episodes was not related to BDNF genotypes but was significantly associated with younger age of onset and with a history of depression in first degree relatives.

Conclusion BDNF genotyping may be informative for anticipating chronicity in major depression. Psychiatry Investig 2013;10:56-61

Key Words Brain-derived neurotrophic factor (BDNF), BDNF Val66Met, Major depressive disorder, Clinical course, Chronicity, Recurrent depression.

\section{INTRODUCTION}

Major depressive disorder (MDD) is often a chronic or recurrent illness with considerable morbidity and mortality. Although the median episode lengths of depressive episodes have been reported to be approximately 3-6 months, a sizeable group of patients report episodes of 24 months or longer. In general, $19.5 \%$ of cases of unipolar depression are chronic. ${ }^{1}$ However, little is known about the genetic factors that may influence the course of MDD.

Current neuroplasticity theories of MDD focus on neuronal atrophy or glial cell death in limbic structures. ${ }^{2}$ Among the fac-

Received: June 18, 2012 Revised: July 16, 2012

Accepted: July 30, 2012 Available online: January 24, 2013

$\triangle$ Correspondence: Doh Kwan Kim, MD, PhD

Department of Psychiatry, Samsung Medical Center, Sungkyunkwan University School of Medicine, 50 Irwon-dong, Gangnam-gu, Seoul 135-710, Republic of Korea

Tel: +82-2-3410-3582, Fax: +82-2-3410-0941, E-mail: paulkim@skku.edu

(a) This is an Open Access article distributed under the terms of the Creative Commons Attribution Non-Commercial License (http://creativecommons.org/licenses/bync/3.0) which permits unrestricted non-commercial use, distribution, and reproduction in any medium, provided the original work is properly cited. tors identified as playing an important role in neuronal plasticity and in the action of antidepressant treatments (e.g., neurogenesis, stabilizing synaptic contacts) is brain-derived neurotrophic factor (BDNF). ${ }^{3}$ Impaired neuronal plasticity could lead to lower responses to antidepressants and lower remission rates, resulting in delayed recovery from MDD.

Accordingly, the BDNF gene appears to be a potential candidate mediator of mechanisms relevant to the pathogenesis of chronic depression. In particular, interest has focused on a common single nucleotide polymorphism (dbSNP reference number rs6265) at nucleotide 196 (G/A), producing an amino acid substitution (valine to methionine) at codon 66 (Val$66 \mathrm{Met}$ ) in the 5 ' pro-BDNF sequence. It has been demonstrated that the Met-BDNF allele is associated with decreased activity-dependent secretion of BDNF in hippocampus and adversely affects grey matter volume in hippocampus and prefrontal cortex. ${ }^{4}$ The Met-BDNF allele also can be associated with various psychiatric disorders including geriatric depression and anxiety. 2,56

If variation in the BDNF genotype is associated with the 
course of MDD, it may be possible to anticipate the course of depression by determining the BDNF genotype at the beginning of treatment. The importance of detecting the course of depression in the early phase lies in the fact that more intensive initial treatment may prevent MDD from taking a chronic course. $^{7-9}$

This study was designed to test for an association between polymorphisms in the BDNF gene and the clinical course of MDD.

\section{METHODS}

\section{Participants}

Two hundred nine patients presenting for treatment of an ongoing depressive episode, who met the Diagnostic and Statistical Manual of the American Psychiatric Association (DSMIV) criteria for MDD, were enrolled in the study. They received treatment in the Department of Psychiatry, Sungkyunkwan University, Seoul, South Korea. A diagnosis of MDD was based on clinical assessment by a board certified psychiatrist, followed by the Structured Clinical Interview for DSMIV Axis I Disorders-Clinical Version (SCID-IV), ${ }^{10}$ which was administered by a trained research assistant. In accordance with our routine clinical procedures, these interviews were conducted with the patient and at least one family member, who provided collateral information. The clinical observations, medical records, past histories, and the DSM-IV criteria were also assessed independently by a second board-certified psychiatrist (DKK). At entry, all patients received an additional semi-structured diagnostic interview, the Samsung Psychiatric Evaluation Schedule (SPES). ${ }^{11}$ The SPES provides information about psychiatric symptoms, comorbid psychiatric diagnoses, and psychosocial variables (age, sex, age of onset, duration of current episode, episode number, family psychiatric history with a focus on mood disorders), and initial severity rating with the Hamilton Depression Rating Scale (HAM-D). ${ }^{12}$ Each patient's medical records were subjected to a retrospective and detailed review to extract demographic data, age of onset, and family history. These reviews were performed without knowledge of the BDNF genotypes. Inclusion criteria were 18 years of age or older, the existence of a unipolar major depressive episode as verified by DSM-III/IV criteria, elapsed time of at least 2 years since first episode onset, and consent for inclusion.

Exclusion criteria in the presenting episode were pregnancy, significant medical conditions, abnormal laboratory baseline values, a history of alcohol or drug dependence, seizure, neurological illness, or concomitant Axis I psychiatric disorder.

One hundred one healthy volunteers with no history of psychiatric illness were recruited by advertisement. Healthy con- trol participants were recruited on the basis of age and sex matching to patients. They were screened by the Minnesota Multiphasic Personality Inventory (MMPI), on which they had validity and clinical scale scores ranging from 35 to $65 .{ }^{13}$ All patients and control subjects are ethnic Koreans.

The protocol was approved by the Institutional review board of Samsung Medical Center, Seoul, South Korea. Signed informed consent was obtained from all participants.

\section{Course of depression}

In keeping with DSM-IV-TR, we defined a chronic major depressive episode as one that is continuously present for at least 2 years. ${ }^{14}$ The recurrence rate may increase with each subsequent episode. ${ }^{15}$ In addition, with each subsequent episode of depression, the length of time to recurrence is shortened. At a certain point, the progressively more rapid time to recurrence in sequential episodes appears to level off, occurring within the third or fourth episode. ${ }^{15}$ Thus, the recurrent group (with three or more previous episodes) has a significantly higher relapse rate after future recoveries. ${ }^{15}$ Based on this evidence, recurrent tendencies in this study were defined as three or more lifetime episodes including the current episode.

\section{BDNF genotyping methods}

Venous blood was drawn into Vacutainer tubes containing EDTA as an anticoagulant. Samples were then prepared into plasma and buffy coat by centrifugation at $3500 \mathrm{rpm}$ for 10 min. Genomic DNA (gDNA, chromosomal DNA) was purified from lymphocytes in the buffy layer using Wizard gDNA purification kit (Promega Inc, Madison, WI, USA).

To examine the BDNF Val66Met polymorphism, an RFLP (Restriction Fragment Length Polymorphism) method was applied. ${ }^{16}$ In brief, the polymorphic region was amplified with primers flanking the exon 2 region of the BDNF gene. These primers were 5'-ACTCTGGAGAGCGTGAAT-3' and 5'ATACTGTCACACACGCTC-3'. The Polymerase Chain Reaction (PCR) reaction mixture consisted of $20 \mathrm{ng}$ gDNA, 0.25 mM dNTP mix, 1x buffer containing $1 \mathrm{mM} \mathrm{MgCl} 2,2.5 \mathrm{mM}$ each of forward and reverse primer, and $0.2 \mathrm{uL}$ of i-MaxII polymerase (iNtRon Biotech, local company in R.Korea). The reaction was performed under the following conditions, one cycle of pre-denaturation at $95^{\circ} \mathrm{C}$ for $5 \mathrm{~min}, 35$ cycles of denaturation at $95^{\circ} \mathrm{C}$ for $30 \mathrm{sec}$, annealing at $57^{\circ} \mathrm{C}$ for $45 \mathrm{sec}$, and elongation at $72^{\circ} \mathrm{C}$ for $45 \mathrm{sec}$ and one cycle of post-elongation at $72^{\circ} \mathrm{C}$ for $10 \mathrm{~min}$. Then, $10 \mathrm{uL}$ amplified $308 \mathrm{bp}$ product was fragmented by incubation with 15 units of the restriction enzyme, NlaIII (NEB Inc., Beverly, MA, USA) at $37^{\circ} \mathrm{C}$ overnight. The restriction fragments were separated on a $3 \%$ agarose gel containing ethidium bromide through electrophoresis at $100 \mathrm{~V}$ for 1 hour and visualized on a UV transluminator. The cutting 


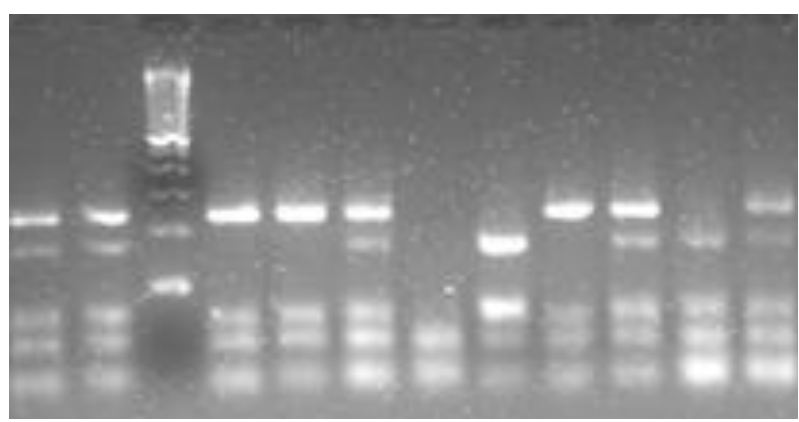

Figure 1. The polymerase chain reaction (PCR) is a biochemical technology in molecular biology to amplify a single or a few copies of a piece of DNA across several orders of magnitude, generating thousands to millions of copies of a particular DNA sequence.

band pattern was $168 \mathrm{bp}+75 \mathrm{bp}+65 \mathrm{bp}$ for the homozygous Methionine (Met)-BDNF allele, $243 \mathrm{bp}+65 \mathrm{bp}$ for the homozygous Valine (Val)-BDNF allele, and $168 \mathrm{bp}+75 \mathrm{bp}+65 \mathrm{bp}+$ $243 \mathrm{bp}$ for the heterozygous Val/Met genotype. Figure 1 shows the cutting band pattern.

\section{Statistical analysis}

For continuous variables, we presented median and interquartile range as descriptive statistics, and the Mann-Whitney test was used for two group comparisons because the variables were not normally distributed. Normality was examined by the Kolmogorov Smirnov test and the Shapiro Wilk test. For categorical variables, frequencies and proportions were presented and the Chi-square test was performed to compare the distributions of the proportions between groups. To investigate gene-dose effect of Met-BDNF allele, the Cochran-Armitage test was applied. Bonferroni's correction was used to control inflated type I error rate due to multiple testing. Deviations from Hardy-Weinberg equilibrium in the patients and control samples for the BDNF Val66Met polymorphisms were investigated. We assumed that the maximum Met/Met homozygote proportion in patients with duration $<24$ month and with $\geq 24$ month was 0.1 and 0.5 , respectively. Power calculation indicated power $>80 \%$ at the 0.05 level of significance. Results were considered as statistically significant at two-sided p-value $<0.05$. All statistical analyses were performed using PASW 17.0.

\section{RESULTS}

\section{Subject characteristics}

Descriptive statistics for clinical and demographic characteristics are shown in Table 1. Among 209 patients, 47 (22.5\%) were male, 162 (77.5\%) were female, and the age range was 22 85 years (median 62, interquartile range 56-67). Healthy control participants comprised 21 (21\%) males and 80 (79\%) females with an age range of 46-77 years (median 61, interqu- artile range 58-64). There was no significant difference between the MDD and healthy control group in age $(\mathrm{p}=0.453)$ and sex $(\mathrm{p}=0.735)$. There was no significant difference in sex, age, age of onset, initial HAM-D scores, or first degree family history between patients with and without chronicity. The age $(\mathrm{p}=0.383)$ and $\operatorname{sex}(\mathrm{p}=0.144)$ distributions between patients with and without recurrent tendencies were not different. However, patients with recurrent tendencies exhibited earlier ages of onset ( 46.5 vs. $56, \mathrm{p}<0.001$ by Mann-Whitney test), higher initial HAM-D score ( 21 vs. 19, $\mathrm{p}=0.033$ by Mann-Whitney test) and higher rate of first degree family history (36\% vs. $12 \%, \mathrm{p}<0.001$ by chi-square test). Patients with a positive first degree family history had a $67 \%$ rate of recurrent illness (31 of 46), whereas patients with a negative first degree family history had a $34 \%$ rate of recurrent illness (55 of 163) (odds ratio 4.06 (2.02-8.14); $\mathrm{p}<0.0001$ by chi-square test).

\section{BDNF genotype and allele distribution in MDD patients and control subjects}

The detailed distributions of the gene variants of the BDNF Val66Met polymorphism, consisting of count and frequency (\%) of genotypes (Met/Met, Met/Val and $\mathrm{Val} / \mathrm{Val}$ ), and of alleles (Met, Val) are shown in Table 2. Among 209 MDD patients, 62 patients $(29.7 \%$ ) had the Val/Val genotype group, 102 (48.8\%) had the Val/Met genotype group and 45 patients (21.5\%) had the Met/Met genotype. Genotype distribution of 101 healthy control subjects was Val/Val (22.8\%), Val/Met (61.4\%) and Met/Met (15.8\%). The distributions of Val66Met genotypes did not differ significantly between patients and healthy controls ( $\mathrm{p}=0.228$ by chi-square test). The Val and Met-BDNF allele distributions also did not differ significantly between the healthy control group and patient group ( $\mathrm{p}=1.000$ by chi-square test).

\section{Chronicity and recurrent tendencies in depressive patients according to BDNF polymorphism}

Patients with Val/Val genotype showed the lowest chronicity rate $(12.9 \%)$ and Met/Met genotype group had the highest chronicity rate (33.3\%). Moreover, there was a gene-dosage effect for Met-BDNF allele on chronicity showing linear relationship between the number of Met-BDNF alleles and chronic depression ( $\mathrm{p}=0.024$ by Cochran-Armitage test). There was a statistically significant difference in chronicity rates ( $27.1 \%$ vs $.16 .8 \%, \mathrm{p}<0.022$ by chi-square test) associated with the Met and Val alleles, respectively (Table 2). Compared with the $\mathrm{Val} / \mathrm{Val}$ genotype, the relative risk of chronicity was 1.67 for the Val/Met genotype, and 2.58 for the Met/Met genotype.

There were no significant associations of BDNF genotype or allele with recurrent course of illness (Table 2). 
Table 1. Characteristics of depressed patient and healthy control group

\begin{tabular}{|c|c|c|c|c|c|c|c|c|c|}
\hline \multirow[t]{2}{*}{ Characteristics } & \multicolumn{3}{|c|}{$\begin{array}{c}\text { Chronicity } \\
\text { (duration of current episode) }\end{array}$} & \multicolumn{3}{|c|}{$\begin{array}{l}\text { Recurrent tendency } \\
\text { (episode number) }\end{array}$} & \multirow{2}{*}{$\begin{array}{c}\text { All } \\
\text { patient }\end{array}$} & \multirow{2}{*}{$\begin{array}{l}\text { Healthy } \\
\text { control }\end{array}$} & \multirow[t]{2}{*}{ p-value ${ }^{\ddagger}$} \\
\hline & $\geq 24$ months & $<24$ months & p-value & $\geq 3$ & $<3$ & $\mathrm{p}$-value & & & \\
\hline Total & 45 & 164 & $0.448^{\dagger}$ & 86 & 123 & $0.144^{\dagger}$ & 209 & 101 & $0.735^{\dagger}$ \\
\hline Male & 12 & 35 & & 15 & 32 & & 47 & 21 & \\
\hline Female & 33 & 129 & & 71 & 91 & & 162 & 80 & \\
\hline Age & $59(49-67)$ & $63(54.5-70)$ & $0.161^{*}$ & $63(54-70)$ & $62(51-68)$ & $0.383^{*}$ & $62(56-67)$ & $61(58-64)$ & $0.453^{*}$ \\
\hline Age of onset & $46(38-60)$ & $52(37.5-60)$ & $0.576^{*}$ & $46.5(36-56)$ & $56(44-61)$ & $<0.001^{*}$ & $52(37.5-60)$ & & \\
\hline Initial HAM-D & $19(17-23)$ & $20(17-24)$ & $0.958^{*}$ & $21(18-24)$ & $19(17-23)$ & $0.033^{*}$ & $20(17-24)$ & & \\
\hline $\begin{array}{l}\text { 1st degree family } \\
\text { history }(\%)\end{array}$ & $6(13.3)$ & $40(24.4)$ & $0.113^{\dagger}$ & $31(36)$ & $15(12.2)$ & $<0.001^{\dagger}$ & $46(22)$ & & \\
\hline
\end{tabular}

${ }^{*}$ Mann-Whitney $\mathrm{U}$ test: value is median (inter-quartile range), ${ }^{\dagger}$ chi-square test: value is the number of patients, ${ }^{\ddagger}$ comparison between the depressed patient and healthy control group. HAM-D: Hamilton Rating Scale for Depression score

Table 2. Brain-Derived Neurotrophic Factor (BDNF) genotype and allele distribution in depressed patient and healthy control group

\begin{tabular}{|c|c|c|c|c|c|c|c|c|c|c|c|}
\hline & \multicolumn{4}{|c|}{ Chronicity (duration of current episode) } & \multicolumn{4}{|c|}{ Recurrent tendency (episode number) } & \multirow{2}{*}{$\begin{array}{c}\text { All patients } \\
(\%)\end{array}$} & \multirow{2}{*}{$\begin{array}{c}\text { Controls } \\
\text { (\%) }\end{array}$} & \multirow[b]{2}{*}{ p-value ${ }^{\dagger}$} \\
\hline & $\begin{array}{c}\geq 24 \\
\text { months }\end{array}$ & $\begin{array}{c}<24 \\
\text { months }\end{array}$ & $\begin{array}{c}\text { Chronicity } \\
\text { rate }(\%)\end{array}$ & p-value & $\geq 3$ & $<3$ & $\begin{array}{c}\text { Recurrent } \\
\text { rate }(\%)\end{array}$ & p-value & & & \\
\hline Genotype & & & & & & & & & & & 0.228 \\
\hline Val/Val & 8 & 54 & 12.9 & $0.024^{*}$ & 29 & 33 & 87.9 & $1.000^{*}$ & $62(29.7)$ & $23(22.8)$ & \\
\hline Val/Met & 22 & 80 & 21.6 & & 38 & 64 & 59.4 & & $102(48.8)$ & $62(61.4)$ & \\
\hline Met/Met & 15 & 30 & 33.3 & & 19 & 26 & 73 & & $45(21.5)$ & $16(15.8)$ & \\
\hline Total & 45 & 164 & 21.5 & & 86 & 123 & 69.9 & & $209(100)$ & $101(100)$ & \\
\hline Allele & & & & & & & & & & & 1.000 \\
\hline Val & 38 & 188 & 16.8 & $0.022^{\ddagger}$ & 96 & 130 & 42.5 & $1.098^{\ddagger}$ & $226(54.1)$ & $108(53.5)$ & \\
\hline Met & 52 & 140 & 27.1 & & 76 & 116 & 39.6 & & $192(45.9)$ & $94(46.5)$ & \\
\hline Total & 90 & 328 & 21.5 & & 172 & 246 & 41.1 & & $418(100)$ & $202(100)$ & \\
\hline
\end{tabular}

The Bonferroni correction is used for multiple comparisons. *Cochran-Armitage test: comparison among the BDNF genotypes to investigate dose dependent effect of Met-BDNF allele, ${ }^{\dagger}$ chi-square test: comparison between the depressed patient and healthy control group, ${ }^{\ddagger}$ chi-square test: comparison between Val-BDNF allele and Met-BDNF allele. Val: valine, Met: methionine

\section{DISCUSSION}

Our study identified an effect of BDNF Val66Met polymorphism on the course of depression in patients with MDD.

The frequency order with respect to genotype was identical between MDD patients and healthy control participants. This result was consistent with other studies involving Asian populations. ${ }^{16-18}$ Therefore, it appears that the Met-BDNF allele does not determine the genetic liability to MDD, but rather predicts the chronicity of MDD.

In the analysis for the relationship between BDNF Val66Met polymorphism and chronicity, we observed a significant dose effect for the Met-BDNF allele's association with higher rates of chronic depression (Table 2).

Patients with recurrent tendencies exhibited an earlier age of onset, higher rate of first degree family history and higher initial HAM-D score (Table 1). Previous studies demonstrated the same result that early age at onset and the positive family history of depression would be significant predictors for a high risk of recurrence. ${ }^{19}$

The Met-BDNF allele adversely affects prefrontal and hippocampal anatomy and functions to reduce grey matter volume in the hippocampus and dorsolateral prefrontal cortex. ${ }^{2}$ Brain imaging studies have demonstrated a reduction in the volume of the hippocampus of depressed subjects. ${ }^{20}$ Thus, decreased levels of BDNF associated with the Met/Met genotype would be consistent with prolonged depressive episodes. Although a cross-sectional study ${ }^{21}$ reported that there is no Val66Met effect on hippocampal volume, these patients were not experiencing chronic depressive episodes.

BDNF also may interact with psychosocial, non-drug factors that moderate response to antidepressant treatments. For instance, it is known that social support moderates responses to medical treatment of depression. ${ }^{22} \mathrm{BDNF}$ is a key regulator of the mesolimbic dopamine pathway that mediates the identification of and response to emotionally salient environmen- 
tal stimuli. ${ }^{23}$

Interestingly, several studies have reported differences in the distribution of BDNF Val66Met polymorphisms according to ethnicity in healthy individuals. The most frequent genotype among Korean healthy participants was Val/Met but Val/Val was most frequent among Caucasian healthy participants. ${ }^{17,24}$ Healthy Japanese also carried less Val/Val genotype than Italians or Americans. ${ }^{17,24}$ These significant ethnic differences may arise from natural selection or from environmental factors. ${ }^{13}$ In light of these ethnic differences in BDNF Val66Met polymorphisms, we limited this study to Korean patients.

Our study assessed clinical course and episode duration retrospectively. Hence, recall bias could have affected both the detection of past episodes and estimates of the duration of illness. Also, in this initial study we did not consider interactions among other genes relevant to depression. There are possible interactions of the BDNF Val66Met polymorphism with other common gene variants, such as serotonin transporter promoter polymorphism (5-HTTLPR) and dopamine transporter gene (DAT VNTR), thought to be involved in the pathophysiology of affective disorders. ${ }^{25}$ Despite this potential limitation, we did observe a strong dose effect of the MetBDNF allele on chronicity.

Another limitation is that we did not screen healthy controls with a structured diagnostic interview. There is a possibility that some control subjects with heavy family histories of mood disorders would later develop depression. However, any such future events would have no effect on the significant differences we observed within the group of patients (Table 2).

We adjusted for age, onset age, family history, sex, and the severity of depression (baseline HAM-D), and excluded the possibility of comorbidity; however, other factors affecting the chronicity of depression like personality, less education, socioeconomic status, unemployment, greater general medical illness burden were not included as cofactors in our analysis. Small sample size of subjects is another limitation of this study.

Nevertheless, for the first time, this study demonstrated a significant possibility that the Met/Met genotype acts as a robust moderator of chronicity of depression.

\section{Conclusion}

Among the BDNF gene polymorphisms, the Met allele showed a significant dosage association with chronicity of depressive episodes. Compared with the Val/Val genotype, the relative risk of chronicity was 1.67 for the Val/Met genotype, and 2.58 for the Met/Met genotype. Knowledge of a patient's Val66Met BDNF genotype, therefore, may signal the need for early intensive treatment aimed at preventing chronic depressions.

\section{Acknowledgments}

This study was supported by grants from the Korea Science and Engineering Foundation (KOSEF) NRL Program (Grant R0A-2007-000-201290 ), Center for Genome Research of Samsung Biomedical Research Institute (GRC-P-10-03), and Samsung Biomedical Research Institute grant (C-A9205). Dr. D.K.Kim reported receiving grant support from Lundbeck. In the past 3 years, Dr. Bernard Carroll has received compensation from AstraZeneca, MultiHealth Systems, South Carolina Psychiatric Association, Springer Publishing Company and Brentwood Biomedical Research Institute, Los Angeles. No other potential conflict of interest relevant to this article was reported.

\section{REFERENCES}

1. Angst J, Preisig M. Outcome of a clinical cohort of unipolar, bipolar and schizoaffective patients. Results of a prospective study from 1959 to 1985. Schweiz Arch Neurol Psychiatr 1995;146:17-23.

2. Frodl T, Schule C, Schmitt G, Born C, Baghai T, Zill P, et al. Association of the brain-derived neurotrophic factor Val66Met polymorphism with reduced hippocampal volumes in major depression. Arch Gen Psychiatry 2007;64:410-416.

3. Duman RS, Monteggia LM. A neurotrophic model for stress-related mood disorders. Biol Psychiatry 2006;59:1116-1127.

4. Egan MF, Kojima M, Callicott JH, Goldberg TE, Kolachana BS, Bertolino A, et al. The BDNF val66met polymorphism affects activity-dependent secretion of BDNF and human memory and hippocampal function. Cell 2003;112:257-269.

5. Chen ZY, Jing D, Bath KG, Ieraci A, Khan T, Siao CJ, et al. Genetic variant BDNF (Val66Met) polymorphism alters anxiety-related behavior. Science 2006;314:140-143

6. Hwang JP, Tsai SJ, Hong CJ, Yang CH, Lirng JF, Yang YM. The Val66Met polymorphism of the brain-derived neurotrophic-factor gene is associated with geriatric depression. Neurobiol Aging 2006;27:1834-1837.

7. Nierenberg AA, Petersen TJ, Alpert JE. Prevention of relapse and recurrence in depression: the role of long-term pharmacotherapy and psychotherapy. J Clin Psychiatry 2003;64(Suppl 15):13-17.

8. Quitkin FM. The importance of dosage in prescribing antidepressants. Br J Psychiatry 1985;147:593-597.

9. Agency for Health Care Policy and Research. Depression Guideline Panel. Clinical Practice Guideline. Number 5: Depression in Primary Care, Volume 2. Treatment of Major Depression. U.S. Dept. of Health and Human Services, Public Health Service, Rockville, MD (1993) AHCPR Publication No. 93-0551 1993.

10. First MB, Gibbon M, Spitzer RL, Williams JBW. Structured Clinical Interview for DSM-IV Axis I Disorders-Clinical Version (SCID-CV). Washington, DC: American Psychiatric Press, Inc; 1997.

11. Kim DK, Lim SW, Lee S, Sohn SE, Kim S, Hahn CG, et al. Serotonin transporter gene polymorphism and antidepressant response. Neuroreport 2000;11:215-219.

12. Hamilton M. A rating scale for depression. J Neurol Neurosurg Psychiatry 1960;23:56-62.

13. Colligan RC, Osborne D, Swenson WM, Offord KP. The MMPI: development of contemporary norms. J Clin Psychol 1984;40:100-107.

14. American Psychiatric Association. Diagnostic and Statistical Manual of Mental Disorders - Text Revision (DSM-IV TR) (4th ed). Arlington, VA: American Psychiatric Association; 2000.

15. Keller MB, Boland RJ. Implications of failing to achieve successful longterm maintenance treatment of recurrent unipolar major depression. Biol Psychiatry 1998;44:348-360.

16. Choi MJ, Kang RH, Lim SW, Oh KS, Lee MS. Brain-derived neurotrophic factor gene polymorphism (Val66Met) and citalopram response in major depressive disorder. Brain Res 2006;1118:176-182.

17. Pivac N, Kim B, Nedic G, Joo YH, Kozaric-Kovacic D, Hong JP, et al. Ethnic differences in brain-derived neurotrophic factor Val66Met polymorphism in Croatian and Korean healthy participants. Croat Med J 
2009;50:43-48.

18. Tsai SJ, Cheng CY, Yu YW, Chen TJ, Hong CJ. Association study of a brain-derived neurotrophic-factor genetic polymorphism and major depressive disorders, symptomatology, and antidepressant response. Am J Med Genet B Neuropsychiatr Genet 2003;123B:19-22.

19. Rush AJ. The varied clinical presentations of major depressive disorder. J Clin Psychiatry 2007;68(Suppl 8):4-10.

20. Videbech P, Ravnkilde B. Hippocampal volume and depression: a meta-analysis of MRI studies. Am J Psychiatry 2004;161:1957-1966.

21. Jessen F, Schuhmacher A, von Widdern O, Guttenthaler V, Hofels S, Suliman $\mathrm{H}$, et al. No association of the Val66Met polymorphism of the brain-derived neurotrophic factor with hippocampal volume in major depression. Psychiatr Genet 2009;19:99-101.
22. Hybels CF, Blazer DG, Steffens DC. Partial remission. A common outcome in older adults treated for major depression. Geriatrics 2006;61: 22-26.

23. Wise RA. Dopamine, learning and motivation. Nat Rev Neurosci 2004; 5:483-494.

24. Shimizu E, Hashimoto K, Iyo M. Ethnic difference of the BDNF 196G/ A (val66met) polymorphism frequencies: the possibility to explain ethnic mental traits. Am J Med Genet B Neuropsychiatr Genet 2004;126B: 122-123.

25. Hunnerkopf R, Strobel A, Gutknecht L, Brocke B, Lesch KP. Interaction between BDNF Val66Met and dopamine transporter gene variation influences anxiety-related traits. Neuropsychopharmacology 2007;32: 2552-2560. 